ORIGINAL ARTICLE

\title{
Crisis management during anaesthesia: hypotension
}

\author{
R W Morris, L M Watterson, R N Westhorpe, R K Webb
}

Qual Saf Health Care 2005;14:e1 1 (http://www.qshc.com/cgi/content/full/14/3/e11). doi: 10.1136/qshc.2002.004440

See end of article for authors' affiliations

.....................

Correspondence to: Professor W B Runciman, President, Australian Patient Safety Foundation GPO Box 400, Adelaide, South Australia 5001 , Australia; research@apsf. net.au

Accepted 11 January 2005
Background: Hypotension is commonly encountered in association with anaesthesia and surgery. Uncorrected and sustained it puts the brain, heart, kidneys, and the fetus in pregnancy at risk of permanent or even fatal damage. Its recognition and correction is time critical, especially in patients with pre-existing disease that compromises organ perfusion.

Objectives: To examine the role of a previously described core algorithm "COVER ABCD-A SWIFT CHECK", supplemented by a specific sub-algorithm for hypotension, in the management of hypotension when it occurs in association with anaesthesia.

Methods: Reports of hypotension during anaesthesia were extracted and studied from the first 4000 incidents reported to the Australian Incident Monitoring Study (AIMS). The potential performance of the COVER $A B C D$ algorithm and the sub-algorithm for hypotension was compared with the actual management as reported by the anaesthetist involved.

Results: There were 438 reports that mentioned hypotension, cardiovascular collapse, or cardiac arrest. In $17 \%$ of reports more than one cause was attributed and 550 causative events were identified overall. The most common causes identified were drugs (26\%), regional anaesthesia (14\%), and hypovolaemia (9\%). Concomitant changes were reported in heart rate or rhythm in $39 \%$ and oxygen saturation or ventilation in $21 \%$ of reports. Cardiac arrest was documented in $25 \%$ of reports. As hypotension was frequently associated with abnormalities of other vital signs, it could not always be adequately addressed by a single algorithm. The sub-algorithm for hypotension is adequate when hypotension occurs in association with sinus tachycardia. However, when it occurs in association with bradycardia, non-sinus tachycardia, desaturation or signs of anaphylaxis or other problems, the sub-algorithm for hypotension recommends cross referencing to other relevant sub-algorithms. It was considered that, correctly applied, the core algorithm COVER ABCD would have diagnosed $18 \%$ of cases and led to resolution in two thirds of these. It was further estimated that completion of this followed by the specific sub-algorithm for hypotension would have led to earlier recognition of the problem and/or better management in $6 \%$ of cases compared with actual management reported.

Conclusion: Pattern recognition in most cases enables anaesthetists to determine the cause and manage hypotension. However, an algorithm based approach is likely to improve the management of a small proportion of atypical but potentially life threatening cases. While an algorithm based approach will facilitate crisis management, the frequency of co-existing abnormalities in other vital signs means that all cases of hypotension cannot be dealt with using a single algorithm. Diagnosis, in particular, may potentially be assisted by cross referencing to the specific sub-algorithms for these.
$\mathrm{H}$ ypotension is commonly encountered during anaesthesia and may be mild and self-limiting. Sustained uncorrected hypotension has the potential to impair organ perfusion to such a degree as to result in irreversible ischaemic damage. When vital organs are involved, the result may be permanent neurological deficit, cardiomyopathy, or renal impairment. In the pregnant patient, the well being of the fetus may be compromised. In the acute setting, profound hypotension may herald or precipitate a cardiac arrest. In patients with limited cardiac reserve, even relatively mild levels of hypotension-to degrees often considered acceptable with "standard" anaesthetic techniques-might be problematic in terms of myocardial ischaemia. This has been exemplified with propofol associated deaths in ASA III-IV patients. ${ }^{1}$ Cardiac reserve may also be reduced in patients with sepsis, renal disease, and major trauma in addition to those with intrinsic myocardial disease.

Hypotension is commonly associated with abnormalities of other vital signs, in particular heart rate and rhythm. Similarly, abnormalities of oxygenation and gas exchange may be present as evidenced by desaturation on pulse oximetry and problems with ventilation. It is important to determine which abnormality represents the primary problem as this will influence the differential diagnosis and management. This issue is worthy of consideration as it presents implications for workload and time management. In many cases hypotension should be adequately managed by recognition of simple patterns and, in such circumstances, a comprehensive elimination of all possible causes in every episode would result in unnecessary treatment and invasive procedures. Evidence from the AIMS reports demonstrates, however, that refractory or unexpected hypotension not infrequently represents a problem, as the cause or required management may lie outside the day-to-day repertoire of the anaesthetist. This lends support to the use of a structured approach when all is not going as expected.

In 1993 a "core" crisis management algorithm represented by the mnemonic COVER ABCD-A SWIFT CHECK (the AB precedes COVER for the non-intubated patient) was proposed as the basis for a systematic approach to any crisis during anaesthesia where it is not immediately obvious what should be done or where actions taken have failed to remedy the situation. ${ }^{2}$ This was validated against the first 2000 incidents reported to the Australian Incident Monitoring Study (AIMS). AIMS is an ongoing study which involves the voluntary anonymous reporting of any unintended incident 


\section{HYPOTENSION}

\section{EMERGENCY MANAGEMENT}

Complete COVER ABCD-A SWIFT CHECK (1)*

Confirm the blood pressure change is real (2)

Don't hesitate to treat as Cardiac Arrest $\rightarrow$ page $38^{* *}$

Inform and interrogate the surgeon

Recheck vaporisers are off (3)

Improve posture: lie flat, elevate legs, if possible

IV fluids: crystalloid bolus $-10 \mathrm{ml} / \mathrm{kg}$, and repeat as necessary

Give vasopressor: metaraminol 0.005-0.01 mg/kg IV bolus (4)

If severe give adrenaline $0.001 \mathrm{mg} / \mathrm{kg} \mathrm{IV} \mathrm{bolus,} \mathrm{that} \mathrm{is} 1 \mathrm{ml}$ of

$1: 10,000$

Followed if necessary by an infusion of adrenaline starting at

$0.00015 \mathrm{mg} / \mathrm{kg} / \mathrm{min}(1 \mathrm{ml} / \mathrm{min}$ of $1 \mathrm{mg}$ in $100 \mathrm{ml})$

If erythema, rash or wheeze is evident go to anaphylaxis page $48^{* *}$

If bradycardic give atropine and go to page $28^{* *}$

If pulseless go to cardiac arrest page $38^{* *}$

If desaturated or cyanosed go to hypoxia page $26^{* *}$

Increase monitoring - ECG, arterial line, CVP

\section{REVIEW AND TREAT PROBABLE CAUSES (5) \\ Hypovolaemia (6)}

Consider: Blood loss, dehydration, diuresis, sepsis.

Ensure: Adequate IV access, fluid replacement, cross match

Drugs (3)

Consider: Induction and inhalational agents, opioids, suxamethonium, anticholinesterases, local anaesthetic toxicity, vancomycin, protamine vasopressor/vasodilator infusion problem, drug ampoule or syringe

error and drugs given by surgeon.

Ensure: Agent ceased, support circulation

Regional Anaesthesia (7)

Consider: Vasodilation, bradycardia, respiratory failure.

Ensure: Volume loading, vasopressors (early adrenaline), airway

support, left lateral displacement during pregnancy.

Surgical Events (8)

Consider: Vagal reflexes, obstructed venous return,

pneumoperitoneum, retractors and position.

Ensure: Surgeon aware.

Cardiopulmonary Problems (9)

Consider: Tension pneumothorax, haemothorax, tamponade,

embolism (gas, amniotic or thrombus), sepsis, myocardial depression

(from drugs, ischaemia, electrolytes, trauma)

Ensure: Review of appropriate pages in manual.

The sub-algorithm forms a facing page of the

Crisis Management Manual ${ }^{17}$.

* Numbers in brackets refer to Notes in the right hand panel.

** Page references refer to the Crisis Management Manual ${ }^{17}$.

\section{FURTHER CARE}

Review and treat probable cause(s)

See precipitating factors, and (3) to (9)

Consider further fluid/drug therapy

Consider invasive haemodynamic monitoring

Arterial pressures

Filling pressures

\section{NOTES:}

It was judged that correct use of the algorithm would have led to earlier recognition of the problem and/or better management in $6 \%$ of 438 relevant incidents reported to AIMS.

(1) COVER ABCD accounted fully for $12 \%$ of hypotension incidents.

(2) Use a sphygmomanometer and auscultate the blood pressure. When using an arterial line, check the zero and calibration.

(3) Drugs were a factor in $26 \%$ of causes (inhalational and intravenous anaesthetic agents $7 \%$ each, opioids $5 \%$, suxamethonium $2 \%$ ). Other drugs implicated included vasodilators, inotropes (pump malfunction), IV local anaesthetics, vancomycin, protamine and dilantin. Vaporiser problems made up $32 \%$ of inhalational agent induced hypotension. $2 \%$ of all drug related hypotension was related to syringe or ampoule errors.

(4) Options include IV boluses of ephedrine $0.05 \mathrm{mg} / \mathrm{kg}$ (especially with pregnancy).

(5) Based on all reported incidents timely and effective management was best achieved by using specific algorithms in $85 \%$ of cases (cardiac arrest $25 \%$, desaturation $21 \%$, bradycardia $31 \%$, anaphylaxis $5 \%$, non sinus tachycardia $3 \%)$. The remaining $15 \%$ required review of further possible specific causes. Multiple causes were involved in $23 \%$ of cases.

(6) Hypovolaemia was a factor in $2 \%$ of cases, but is often unreported. It is such a common cause of hypotension that it is not usually reported as an incident.

(7) Regional anaesthesia was a factor in $14 \%$ of cases.

(8) Surgical events were a factor in at least $5 \%$ of cases. Reflex stimulation was induced by anaesthetic procedures (laryngoscopy, CVC placement) as well as surgical manipulations (mesenteric traction, eye surgery).

(9) Cardiopulmonary problems were a factor in $6 \%$ of cases.

These notes comprise a reverse side of a page of the

Crisis Management Manual17.

Figure 1 Hypotension.

which reduced or could have reduced the safety margin for the patient. ${ }^{3}$

It was concluded that, if this algorithm had been correctly applied, a functional diagnosis would have been reached within 40-60 seconds in 99\% of applicable incidents, and the learned sequence of actions recommended by the COVER portion would have led to appropriate steps being taken to handle the $60 \%$ of problems relevant to this portion of the algorithm. ${ }^{2}$ However, this study also showed that the $40 \%$ of problems represented by the remainder of the algorithm ABCD-A SWIFT CHECK were not always promptly diagnosed or appropriately managed. ${ }^{2-4}$ It was decided that it would be useful for these remaining problems to develop a set of subalgorithms in an easy-to-use crisis management manual. ${ }^{5}$ This study reports on the potential place of the COVER ABCD-A SWIFT CHECK algorithm in the diagnosis and initial management of hypotension, provides an outline of a specific crisis management sub-algorithm for hypotension during anaesthesia, and provides an indication of the potential value of using this structured approach.

\section{METHODS}

Of the first 4000 incidents reported to AIMS, those which made reference to hypotension or cardiac arrest were extracted and analysed with respect to the following criteria: the surgical group, anaesthetic technique, patient's ASA status, phase of anaesthesia at the time of presentation, presenting features (including cardiac rhythm, blood pressure, oxygen saturation and other associated vital signs), the presumed precipitating cause, management, and outcome. The COVER ABCD-A SWIFT CHECK algorithm was applied to each report to determine the stages at which the problem might have been detected, and whether the cause would have been diagnosed and the problem resolved by applying the 


\begin{tabular}{|c|c|}
\hline Procedural group & $\begin{array}{l}\text { No of cases of } \\
\text { hypotension }\end{array}$ \\
\hline General surgery & 101 \\
\hline Orthopaedics & 74 \\
\hline Vascular surgery & 43 \\
\hline Urology & 34 \\
\hline Cardiothoracic surgery & 31 \\
\hline Otorhinology & 28 \\
\hline Neurosurgery & 22 \\
\hline Gynaecology & 22 \\
\hline Ophthalmology & 21 \\
\hline Plastic surgery & 18 \\
\hline Obstetrics & 14 \\
\hline Dental surgery & 8 \\
\hline Cardioversion & 3 \\
\hline Diagnostic procedure & 3 \\
\hline Pain management & 3 \\
\hline Electroconvulsive therapy & 1 \\
\hline Other & 2 \\
\hline Not specified & 10 \\
\hline Total & 438 \\
\hline
\end{tabular}

COVER portion of this algorithm. A specific sub-algorithm for hypotension was developed (fig l) and its effectiveness was tested for each report. This was compared with that of the actual management as recorded in each report. In addition to determining a difference between the two approaches with respect to the likelihood of correct diagnosis and management, it was of particular interest during this part of the exercise to evaluate if delays in diagnosis and overall outcome could have been improved with the application of the algorithm.

\section{RESULTS}

There were 438 reports that mentioned the words "hypotension", "cardiovascular collapse" or "cardiac arrest". These occurred across a range of procedural groups (table 1).

The patients' ASA grades were available in 422 of the 434 cases (table 2).

Hypotension was detected during all phases of anaesthesia but most commonly during the induction and maintenance phases (table 3 ).

\section{Aetiology}

In $17 \%$ of cases more than one cause was implicated and 550 causative events were identified in the 438 reports (table 4).

Drugs were considered to have contributed towards hypotension in 144 causative events (26\%) (table 5). Drugs were nominated as causative if they were administered at or just before the hypotensive episode. Reports commonly cited more than one drug, particularly if the hypotension was attributed to induction agents. All induction agents given were nominated in these cases as it was not possible to implicate any particular drug as being responsible. The most commonly implicated drugs were intravenous induction

Table 2 ASA classification

\begin{tabular}{lcc}
\hline ASA grade & No $(\%)$ adults & No $(\%)$ children* \\
\hline I & $91(22 \%)$ & $14(56 \%)$ \\
II & $122(30 \%)$ & $3(12 \%)$ \\
III & $133(33 \%)$ & $5(20 \%)$ \\
IV & $41(10 \%)$ & $2(8 \%)$ \\
V & $10(2 \%)$ & $1(4 \%)$ \\
Not specified & $12(3 \%)$ & $0(0 \%)$ \\
Total & 409 & 25 \\
\hline \multirow{2}{*}{${ }^{*}$ Children aged $<14$ years. }
\end{tabular}

Table 3 Phase of anaesthesia at detection of hypotension

\begin{tabular}{lc}
\hline Phase & $\%$ \\
\hline Pre-induction & 5 \\
Induction & 20 \\
Maintenance & 59 \\
Emergence & 4 \\
Postoperative & 5 \\
Recovery & 3 \\
Not specified & 4 \\
Total & 100 \\
\hline
\end{tabular}

agents in 36 cases, and inhalational agents in 37 cases, 12 of which represented errors with vaporisers. Syringe or ampoule swaps were reported in eight cases.

Hypovolaemia was reported in 50 cases (9\%) and included revealed bleeding $(\mathrm{n}=38)$, occult bleeding from intravascular lines and under drapes $(n=4)$ and dehydration $(n=5)$.

Regional anaesthesia was implicated as a cause on 77 occasions (14\%); it was nominated as a cause whenever it was both present and could have either contributed to the initial hypotension or created difficulty with resuscitation $(17 \%)$. Regional anaesthesia included epidural $(n=34)$, spinal $(\mathrm{n}=36)$, caudal $(\mathrm{n}=1)$, and brachial plexus block anaesthesia $(n=1)$. Combined spinal epidural (CSE) anaesthesia was the technique used in 10 cases of hypotension attributable to neuraxial anaesthesia. Hypotension was attributed to sympathetic blockade in 36 cases and to a high sensory level in 14. Total spinal blockade was reported in six cases. Other mechanisms implicated in causing hypotension included neuraxial opioids $(n=4)$, inadvertent intravenous local anaesthetic $(n=2)$, gas embolism $(n=1)$, and epidural adrenaline $(n=1)$. Caesarian section accounted for six of these which represented nearly half of all cases of obstetric related hypotension $(\mathrm{n}=14)$.

In the $17 \%$ of reports in which more than one cause was identified, the most common combination was hypovolaemia and neuraxial anaesthesia. Hypotension in the presence of epidural or spinal blockade was associated with hypovolaemia in 15 cases, drugs in nine, surgical events in seven, and posture in two. There appeared to be a relationship between hip surgery and spinal or epidural anaesthesia causing hypotension at the time of reduction, reaming or insertion of cement in seven cases, of which three were associated with cardiac arrest.

\section{Diagnosis}

Hypotension was reported without an abnormality of heart rate or rhythm in 158 cases $(36 \%)$. In the remaining cases, hypotension was associated with either cardiac arrest in 108 cases $(25 \%)$, bradycardia or bradydysrhythmia in 134 cases $(31 \%)$, or tachycardia or tachydysrhythmia in 38 cases $(9 \%)$. Desaturation or an abnormality in ventilation was reported in 92 cases $(21 \%)$. Sub-algorithms have been developed for each of these presentations and the diagnosis and management may be expedited by crossing to the relevant sub-algorithm when appropriate. These subgroups were inspected individually and compared with each other and with all cases of hypotension, bradycardia and tachycardia with respect to causes to determine if these revealed any patterns which might be useful in the development of the sub-algorithms, or for guidance on best practice when more than one problem occurred simultaneously (table 4).

An examination of the causes of hypotension associated with bradycardia when compared with the whole bradycardia dataset showed similarities (table 4), although airway problems resulted more often in bradycardia without 
Table 4 Causes of tachycardia, bradycardia and hypotension

\begin{tabular}{|c|c|c|c|c|c|c|}
\hline & Tachy & Brady & Нуро & $\mathrm{H}-\mathrm{CA}$ & H-brady & H-tachy \\
\hline No of reports & 145 & 265 & 438 & 108 & 134 & 38 \\
\hline No of different causes & 148 & 319 & 550 & $\dagger$ & $\dagger$ & 48 \\
\hline \multicolumn{7}{|l|}{ Causes* } \\
\hline Allergy & 11 & 2 & 7 & 7 & 3 & 21 \\
\hline Airway & 4 & 16 & 4 & 5 & 7 & 4 \\
\hline Hypovolaemia & 3 & 3 & 9 & 9 & 4 & 4 \\
\hline Regional anaesthesia & 1 & 9 & 17 & 14 & 16 & 4 \\
\hline Drugs & 33 & 28 & 26 & 26 & 30 & 19 \\
\hline Cardiopulmonary & 8 & 5 & 5 & 6 & 4 & 11 \\
\hline Reflexes & 9 & 14 & 6 & 8 & 14 & 4 \\
\hline Posture & 0 & 1 & 1 & 1 & 1 & 0 \\
\hline Sepsis & 1 & 2 & 1 & 1 & 1 & 0 \\
\hline Surgery§ & 6 & 4 & 2 & 4 & 2 & 6 \\
\hline Pre-existing condition & 1 & 3 & 1 & 2 & 3 & 0 \\
\hline $\mathrm{CNS}^{* *}$ & 1 & 1 & 0 & 0 & 0 & 0 \\
\hline Metabolic†† & 2 & 3 & 1 & 1 & 2 & 0 \\
\hline Monitoring & 1 & 1 & 2 & 2 & 1 & 4 \\
\hline Light anaesthesia & 4 & 0 & 0 & 0 & 0 & 0 \\
\hline Hypercapnia & 4 & 0 & 0 & 0 & 0 & 0 \\
\hline Other & 2 & 0 & 1 & 1 & 0 & 6 \\
\hline Not determined & 8 & 8 & 16 & 13 & 11 & 17 \\
\hline Total $(\%) \ddagger$ & 99 & 100 & 99 & 101 & 99 & 100 \\
\hline
\end{tabular}

Tachy, all cases of tachycardia/tachydysrhythmia; ${ }^{7}$ Brady, all cases of bradycardia; ${ }^{6}$ Hypo, all cases of hypotension; H-CA, cardiac arrest in association with hypotension; $\mathrm{H}$-brady, bradycardia in association with hypotension; $\mathrm{H}$-tachy, tachycardia in association with hypotension.

*Percentage of set or subset.

†Data mislaid, causes approximately $20 \%$ greater than reports.

‡Variation from $100 \%$ reflects rounding of percentages.

Examples of causes expressed as number of cases:

- Cardiopulmonary causes: H-CA: myocardial infarct $(n=3)$, gas embolism $(n=1)$, bronchopleural fistula $(n=1)$, pacemaker malfunction $(n=1)$, pressure on pericardium ( $n=2)$; Hypo: myocardial infarct $(n=3)$, myocardial ischaemia $(n=1)$, left ventricular failure $(n=2)$, dysrhythmia $(n=1)$, tension pneumothorax $(n=4)$, haemothorax $(n=2)$, gas embolism $(n=5)$, fat embolism $(n=1)$; H-tachy: left ventricular failure $(n=1)$, tension pneumothorax $(n=2)$, haemothorax $(n=1)$, gas embolism $(n=1)$.

§Surgical causes: H-CA: central venous cannula wire $(n=2)$, hip cement or reaming $(n=3)$, pneumoperitoneum $(n=4)$, tourniquet $(n=1)$, suction $(n=1)$; Hypo: central venous cannula wire $(n=3)$, hip cement or reaming $(n=2)$, pneumoperitoneum $(n=2)$, clamp removal $(n=1)$, inferior vena caval compression $(n=1)$, retractors $(n=1)$, removal of atrial shunt $(n=1)$; H-tachy: central venous cannula wire $(n=2)$, hip cement or reaming $(n=1)$.

${ }^{* *}$ Central nervous system causes: CA: raised intracranial hypertension $(n=1)$;

††Metabolic causes: CA: acidosis $(n=2)$, anaemia $(n=1)$.

Table 5 Drug related causes of hypotension in presentation groups ${ }^{*}$

\begin{tabular}{lcccc}
\hline Drugs & H-CA & H-tachy & H-brady & H-normal \\
\hline IV anaesthetic agents & 9 & 1 & 6 & 20 \\
Opioids & 4 & 0 & 9 & 12 \\
Benzodiazepines & 2 & 1 & 0 & 7 \\
Inhalational agents & 8 & 0 & 23 & 6 \\
Vaporiser incident & 0 & 1 & 2 & 9 \\
Suxamethoniumt & 8 & 0 & 3 & 0 \\
Muscle relaxants & 3 & 0 & 2 & 1 \\
Anticholinesterase & 2 & 0 & 0 & 0 \\
Local anaesthetic & 3 & 1 & 0 & 3 \\
Protamine & 0 & 0 & 0 & 1 \\
Sodium nitroprusside, GTN $¥$ & 0 & 0 & 1 & 5 \\
Vasopressors & 0 & 0 & 2 & 0 \\
Adrenaline/cocaine & 0 & 1 & 0 & 2 \\
Inotropes & 0 & 1 & 0 & 4 \\
Salbutamol & 0 & 1 & 0 & 0 \\
Clonidine withdrawal & 0 & 0 & 1 & 1 \\
Sudafed & 0 & 0 & 0 & 0 \\
Atropine & 1 & 2 & 0 & 0 \\
Miscellaneous & 6 & 1 & 1 & 5 \\
Drug syringe or ampoule & 0 & 1 & 3 & 4 \\
error\$ & & & & \\
Total in each column & 46 & 11 & 53 & 80 \\
\hline
\end{tabular}

$\mathrm{H}-\mathrm{CA}$, cardiac arrest associated with hypotension; $\mathrm{H}$-tachy, tachycardia in association with hypotension; $\mathrm{H}$-brady, bradycardia in association with hypotension; $\mathrm{H}$-normal, hypotension in association with a normal heart rate.

${ }^{*}$ Combinations of drugs were used in some cases.

†Three cases involved suxamethonium hyperkalaemia. \#GTN, glyceryltrinitrate.

- Vancomycin $(n=3), \beta$-blockers $(n=1), \alpha$-blockers $(n=1)$, digoxin $(n=1)$, hydrallazine $(n=1)$, phenytoin $(n=1)$, methylprednisone $(n=1)$. §Thrombostat $(n=1)$, suxamethonium $(n=2)$, neostigmine $(n=2)$, metaraminol $(n=1)$, morphine $(n=1)$, fentanyl $(n=1)$. hypotension, and hypotension was associated with bradycardia more often than pure bradycardia in association with regional anaesthesia. This suggests that cross referencing with the bradycardia sub-algorithm may assist in the diagnosis of hypotension associated with bradycardia. Each algorithm was inadequate if used in isolation. With respect to management, the requirement for atropine is higher in bradycardia related hypotension than in hypotension overall (see the companion paper in this series on bradycardia ${ }^{6}$ ). The cases of tachycardia and tachydysrhythmias alone or in association with hypotension were also compared (table 4) but only a small number of cases was reported. No major

Table 6 Outcome (\% of subset in each outcome category)

\begin{tabular}{lll}
\hline Outcome* $^{*}$ & $\begin{array}{l}\text { Hypo-CA } \\
(\mathbf{n}=\mathbf{1 0 8})\end{array}$ & $\begin{array}{l}\text { Hypo } \\
(\mathbf{n}=\mathbf{4 3 8})\end{array}$ \\
\hline Good & 51 & 66 \\
Death on table & 11 & 3 \\
Death postoperative & 11 & 5 \\
ICU admission & 18 & 8 \\
Coronary care & 0 & 1 \\
HDU admission & 0 & 1 \\
Cardiac event & 4 & 5 \\
Neurological event & 0 & 2 \\
Delayed recovery & 0 & 1 \\
Othert & 1 & 2 \\
Not specified & 5 & 11 \\
\hline
\end{tabular}

Hypo-CA, all cases of cardiac arrest associated with hypotension; Hypo, all cases of hypotension.

*More than one outcome category may apply in each case; columns total over $100 \%$.

†Other: awareness, coagulopathy, aspiration, abandonment of surgery. 
differences were observed. The same conclusion was reached in comparing sinus and non-sinus tachycardia, with the exception of allergy which was associated with sinus tachycardia (see companion paper in this series on tachy(ardia $\left.{ }^{7}\right)$.

Of 108 cases describing cardiac arrest, 40 of these involved degeneration from initial hypotension to a pulseless state. This distinction was made in order to identify diagnostic or management factors which may prevent deterioration from hypotension into cardiac arrest and hence improve outcome. No factors emerged which differentiated the two situations. Overall, $62 \%$ of cardiac arrests involved a bradyarrhythmia. Tachyarrhythmias and asytole were equally represented among the remaining cases (see companion papers on bradycardia $^{6}$ and tachycardia ${ }^{7}$ for a discussion of rhythm). The expected higher incidence of high ASA grades in cardiac arrest was not realised. All grades were represented in the cardiac arrest group.

There were 92 cases $(21 \%)$ of hypotension in which difficulty with ventilation without desaturation was described (airway obstruction, decreased compliance, hypoventilation (20 cases). There was one case of cyanosis and 70 of desaturation on the pulse oximeter, of which 21 represented an artefact resulting from diminished peripheral perfusion. On examining the patterns of vital signs when hypotension was initially detected, it was shown that artefact could not be reliably distinguished from those cases in which real abnormalities existed. It was concluded that the airway and ventilation must be formally assessed even when the saturation is within normal limits or artefact is suspected; in 12 reports airway abnormalities and hypotension co-existed as two separate unrelated problems.

\section{Management}

Management strategies were reported in 356 cases. Management was assessed as conventional or non-conventional on the basis of contemporary teaching. ${ }^{89}$ Nonconventional management was reported in 12 cases. In most cases this represented treatments which have recently been revised-for example, the use of calcium chloride in cardiac arrest $(n=5)^{8}$ and isoprenaline for bradycardia $(n=4) .{ }^{9}$

\section{Outcomes}

A poor outcome was reported in $28 \%$ of cases (table 6 ). Cases were considered to have had a poor outcome if they were not resolved by the completion of the normal recovery period. An exception to this was electrocardiographic evidence of myocardial ischaemia occurring intraoperatively or in the recovery ward. These are included in cardiac events dealt with elsewhere in this set of papers. ${ }^{10}{ }^{11}$

Factors contributing to a poor outcome were evaluated with respect to ASA grade, presentation, cause, and management. As expected, a higher proportion of cases describing a poor outcome occurred in the cardiac arrest group than in those with hypotension alone (table 6). Death associated with hypotension without cardiac arrest was confined to patients with ASA grades III-V. Other causes of morbidity occurred in all ASA grades. There were 13 deaths on the operating table and 21 postoperative deaths. Approximately two thirds of these were associated with a bradyarrhythmia. Cardiovascular and chronic renal diseases were the most common chronic conditions reported and hypovolaemia, sepsis, and acute cardiac dysfunction were commonly reported acute conditions. A relationship between nonconventional treatment and poor outcome was sought but this was not found because the number of reports describing non-conventional treatment was small and because it was difficult to establish a case for preventable morbidity on the basis of the reports alone.

\section{Paediatrics}

Hypotension was described in 25 children under the age of 14 years. Bradycardia was associated in 21 cases, which included nine cardiac arrests. All cases of cardiac arrest involved bradycardia or bradydysrhythmia. The causes attributed were consistent with bradycardia related hypotension overall. A poor outcome was reported in eight cases. These included three deaths on the table and one postoperative death. Significant cardiac or neurological disease was present in all children who died. The remaining four cases involved an ICU admission.

\section{Verification of the sub-algorithm}

When the COVER ABCD-A SWIFT CHECK was applied to each report it was considered that the problem would have been detected in all cases either at the Cl stage of COVER or at $\mathrm{C}$ of $\mathrm{ABCD} ; 18 \%$ of cases $(n=77)$ would have been diagnosed and, of these, two thirds would also have been resolved by application of the core algorithm COVER. The majority of these dealt with vaporiser incidents V2 $(n=9)$ breathing circuit problems Vl $(n=20)$, artefact from monitors Rl $(n=12)$, or equipment malfunction or errors with non-anaesthetic drugs $\mathrm{R} 2(\mathrm{n}=34)$. The remaining cases required either completion of the sub-algorithm for hypotension or pursuit of sub-algorithms for specific incidents dealt with under ABCD-A SWIFT CHECK. It was considered that the completion of the algorithm COVER ABCD-A SWIFT CHECK followed by the sub-algorithm for hypotension shown in fig 1 would have constituted appropriate management in all but six cases ( $1 \%)$. The reasons for the inadequacy of this sub-algorithm in these remaining cases included patient factors (two patients had asymmetrical pulses), problems with monitors (CVP monitors placed in arteries or over-reading), and logistical problems such as delays in receiving blood products.

The effectiveness of this structured approach was compared with that of the actual management as documented by each of the reports, and it was considered that, properly applied, it would have led to quicker and/or better resolution of the problem in 26 cases (6\%). There was a significant delay in diagnosis in 21 of these and incomplete management in five. The most common inadequacies included failure to consider multiple causes-in particular, co-existing hypovolaemia, failure to set monitors appropriately (alarms disabled or left in manual mode), failure to check vaporiser settings or contents, and delays in diagnosing or administering adrenaline in anaphylaxis.

While it was considered that $6 \%$ of cases could have been managed better, it was felt that the reports did not provide adequate information from which to draw conclusions about preventable death and morbidity.

\section{DISCUSSION}

The requirements of the algorithm were considered to be the following:

- To facilitate respiratory and circulatory support as a temporising measure while the diagnosis is considered and definitive treatment is initiated. This would help to prevent degeneration into an unrecoverable condition.

- To promote a tiered approach to blood pressure support, beginning with fluids and posture and upgrading to vasopressors and inotrope therapy, depending on the response.

- To ensure that supportive therapy is simple and based on conventional teaching.

- To ensure that differential diagnosis and definitive management advice is comprehensive enough to cover the wider scope of the problems encountered. 
- To facilitate multiple causes and abnormalities of multiple systems being managed simultaneously to avoid focusing on one system to the exclusion of another. This is particularly relevant to the frequent association between hypotension and desaturation. Considering the common association of respiratory events in the causation or sequelae of end organ hypoperfusion, it was considered mandatory to ensure early aggressive support of both cardiovascular and respiratory systems.

The valuable role of pattern recognition in dealing with clinical problems was evident from the reports, the majority being resolved quickly and simply. However, in $6 \%$ of cases hypotension was reported as prolonged and difficult to manage either because the diagnosis or appropriate treatment was not immediately apparent. Contributing factors were identified and a conclusion made that these delays could have been avoided by the application of a structured management approach to these difficult refractory cases.

The sub-algorithm for hypotension is shown in fig 1 . The hallmarks of the algorithm are early aggressive support of the circulation as a temporising measure while the diagnosis is considered and definitive measures are initiated. Supportive therapy is consistent with contemporary teaching. It begins with simple measures including the COVER algorithm, postural control and fluid therapy, with more aggressive therapy if the response is inadequate. The latter includes vasopressors (phenylephrine, metaraminol, ephedrine) and inotropes (adrenaline). The use of invasive cardiovascular monitoring should also be considered at this point. Definitive treatment is context specific.

Hypotension is frequently associated with abnormalities in other vital signs and it is unlikely that these will be addressed in a single algorithm. Rather, the algorithm for hypotension must succeed in directing the user along specific paths that facilitate identifying the differential diagnosis and appropriate management strategies. It was concluded that, when hypotension occurs in association with bradycardia, nonsinus tachycardia, real or apparent desaturation or signs of anaphylaxis (erythema, bronchospasm) or any other problem, then management would be most expeditious if these are considered to be the primary problem. Pursuing the specific sub-algorithms for these events (presented elsewhere in this series of articles ${ }^{5-7}{ }^{10-13}$ ) will offer definitive as well as supportive management and avoid delays. When hypotension occurs in association with sinus tachycardia, hypotension should be treated as the primary abnormality and the supportive therapy outlined above should be initiated immediately.

One of the most important considerations in the management of hypotension is a prompt response. It is imperative to avoid delays that could include the faithful consideration of an overly comprehensive differential diagnosis. While the hypotension persists at potentially dangerous levels, "confirmation bias" has been identified as one reason why wrong diagnoses are made during crises. ${ }^{514}$ Several issues arose which are worthy of discussion:

- Multiple diagnoses: $17 \%$ of cases involved multiple causesfor example, if spinal or epidural anaesthesia is present then the contribution of posture and hypovolaemia should be specifically addressed.

- Relative drug overdose: "standard treatment" being poorly tolerated because of pre-existing patient condition. Hypotension which appeared to be drug induced with a "standard" dose was called relative overdose. Of the total of 144 cases in which drugs contributed to hypotension, 73 were thought to have involved relative overdoses. This is similar to previous experience with cardiac arrest during

\section{Key messages}

- Among the first 4000 incidents reported to AIMS there were 438 reports $(11 \%)$ describing 550 causative events that identified hypotension during anaesthesia.

- The most commonly affected ASA grades were II and III and the most commonly involved anaesthesia phases were induction and maintenance.

- Drugs $(26 \%)$ and regional anaesthesia (17\%) were cited as the most common causes of tachycardia in this series. These two causes also predominated in the cardiac arrests that occurred in association with hypotension.

- Intravenous anaesthetic agents, opioids, and vaporiser incidents were the most common drug related causes.

- Predominant regional anaesthesia causes of neuraxial hypotension were subarachnoid spinals $(46 \%)$ and epidurals $(44 \%$, including combined spinal epidural blocks 13\%).

- Neuraxial hypotension from the 77 regional blocks was attributed to sympathetic blockade in 36 cases and high sensory level blocks in 14 cases.

- In the $17 \%$ of cases where more than one cause was identified, the most common combination was hypovolaemia and neuraxial anaesthesia.

- No abnormal heart rate or rhythm was reported in 158 cases of hypotension (36\%).

- In $108(25 \%)$ of all the cases of hypotension there was a cardiac arrest. There were no identifiable diagnostic or management factors found that would have enabled the prevention of the 40 cases that degenerated from initial hypotension to a pulseless state.

- Of the 356 cases where management was reported, "non-conventional" management on the basis of contemporary teaching was reported in 12 .

- Poor outcome was reported in $28 \%$ of all cases. There were 34 deaths.

- There were 25 reports involving children under 14 years of age. Bradycardia was associated in 21 of these with nine cardiac arrests; all arrests involved bradycardia or bradyarrhythmia.

- It was considered that, while $12 \%$ of cases would have been appropriately managed with the core algorithm, the remainder required either use of the hypotension sub-algorithm or of other specific sub-algorithms for specific incidents dealt with under $A B C D-A$ SWIFT CHECK.

anaesthesia in which relative overdose was thought to have been responsible for approximately $40 \%$ of drug related events. ${ }^{15}$ In this series of AIMS reports approximately half the reports of relative overdoses involved induction agents $(n=35)$. Propofol was the specified agent in nine cases and thiopentone in 12. In many cases multiple agents were used during induction including fentanyl and midazolam. Only half of all cases of relative overdose $(\mathrm{n}=34)$ occurred in patients of ASA grades III$\mathrm{V}$. Within these grades the most commonly cited preexisting conditions were cardiac failure $(n=11)$, renal impairment $(n=5)$, pulmonary compromise $(n=4)$, advanced age $(\mathrm{n}=3)$, anaemia $(\mathrm{n}=4)$, hypovolaemia $(\mathrm{n}=4)$, cachexia $(\mathrm{n}=2)$, obesity $(\mathrm{n}=1)$, multi-trauma $(\mathrm{n}=1)$, and an immunocompromised state $(\mathrm{n}=1)$. 
- Neuraxial blockade: an association between neuraxial blockade and hypotension was also observed. While the total number of cases was small, there seemed to be a high incidence of cases involving hip surgery when compared with other procedures. This is consistent with the trend observed by the New South Wales Special Committee Investigating Deaths under Anaesthesia in the previous decade. ${ }^{16}$ In the AIMS cases hypotension was often precipitated by reaming or cementing of the femur or acetabulum.

- Concomitant respiratory problems: it is common for an abnormality of airway and ventilation vital signs to coexist with hypotension. This may represent artefact secondary to hypoperfusion of the peripheries or it may be an expression of the same event causing hypotension or exist as a separate event. The actual cause may not be readily apparent and the situation warrants a systematic evaluation.

\section{CONCLUSION}

Hypotension is a common intraoperative event and is usually promptly assessed and successfully managed. However, the scope of hypotension is too large to be confidently managed on every occasion from either pattern recognition or first principles. An algorithm based approach can facilitate diagnosis and management but this cannot be achieved using a single algorithm. Rather, a working directory (or cross referencing system) that diverts the user along high yield diagnostic and management pathways is required. The approach described in fig 1 should support adequate respiratory and cardiovascular function while directing the anaesthetist to appropriate specific sub-algorithms.

\section{ACKNOWLEDGEMENTS}

The authors would like to thank all the anaesthetists in Australia and New Zealand who contributed to the 4000 incident reports upon which this and the other 24 papers in the Crisis Management Series are based. The coordinators of the project also thank Liz Brown for preparing the draft of the original Crisis Management Manual; Loretta Smyth for typing; Monika Bullock RN for earlier coding and classifying of data; Dr Charles Bradfield for the electronic version of the algorithms; Dr Klee Benveniste for literature research; and Drs Klee Benveniste, Michal Kluger, John Williamson and Andrew Paix for editing and checking manuscripts.

\section{Authors' affiliations}

R W Morris, Director, Research and Development, Sydney Medical Simulation Centre, Royal North Shore Hospital, St Leonards, New South Wales, Australia
L M Watterson, Senior Staff Specialist and Director, Sydney Medical Simulation Centre, Royal North Shore Hospital, St Leonards, New South Wales, Australia

R N Westhorpe, Deputy Director, Department of Paediatric Anaesthesia and Pain Management, Royal Children's Hospital, Parkville, Victoria, Australia

R K Webb, Senior Staff Specialist, Department of Anaesthesia and Intensive Care, The Townsville Hospital, Douglas, Queensland, Australia

This study was coordinated by The Australian Patient Safety Foundation, GPO Box 400, Adelaide, South Australia 5001, Australia.

\section{REFERENCES}

1 Warden JC, Pickford DR. Fatal cardiovascular collapse following propofol induction in high-risk patients and dilemmas in the selection of a short-acting induction agent. Anaesth Intensive Care 1995;23:485-7.

2 Runciman WB, Webb RK, Klepper ID, et al. Crisis management: validation of an algorithm by analysis of 2000 incident reports. Anaesth Intensive Care 1993;21:579-92.

3 Webb RK, Currie M, Morgan CA, et al. The Australian Incident Monitoring Study: an analysis of 2000 incident reports. Anaesth Intensive Care 1993;21:520-8.

4 Webb RK, van der Walt JH, Runciman WB, et al. Which monitor? An analysis of 2000 incident reports. Anaesth Intensive Care 1993;21:529-42.

5 Runciman WB, Kluger MT, Morris RW, et al. Crisis management during anaesthesia: the development of an anaesthetic crisis management manual. Qual Saf Health Care 2005; 14:e1.

6 Watterson LM, Morris RW, Westhorpe RN, et al. Crisis management during anaesthesia: bradycardia. Qual Saf Health Care 2005; 14:e9.

7 Watterson LM, Morris RW, Williamson JA, et al. Crisis management during anaesthesia: tachycardia. Qual Saf Health Care 2005; 14:e10.

8 Advanced Life Support Committee, Australian Resuscitation Council. Adult cardio-respiratory arrest algorithm, July 2002 and paediatric cardiorespiratory arrest algorithm, March 2004. Available from: www.resus.org.au (accessed 26 September 2004).

9 Emergency Cardiac Care Committee and Subcommittees, American Heart Association. Guidelines for cardiopulmonary resuscitation and emergency cardiac care. Part III: Adult advanced cardiac life support. 1992 National Conference on Cardiopulmonary Resuscitation (CPR) and Emergency Cardiac Care (ECC). JAMA 1992;268:2199-241.

10 Ludbrook GL, Webb RK, Currie M, et al. Crisis management during anaesthesia: myocardial ischaemia and infarction. Qual Saf Health Care 2005; 14:e13.

11 Runciman WB, Morris RW, Watterson LM, et al. Crisis management during anaesthesia: cardiac arrest. Qual Saf Health Care 2005; 14:e14.

12 Szekely SM, Runciman WB, Webb RK, et al. Crisis management during anaesthesia: desaturation. Qual Saf Health Care 2005; 14:e6.

13 Currie M, Kerridge R, Bacon AK, et al. Crisis management during anaesthesia: anaphylaxis and allergy. Qual Saf Health Care 2005; 14:e19.

14 Runciman WB, Sellen A, Webb RK, et al. Errors, incidents and accidents in anaesthetic practice. Anaesth Intensive Care 1993;21:506-19.

15 Keenan RL, Boyan CP. Cardiac arrest due to anaesthesia. A study of incidence and causes. JAMA 1985;253:2373-7.

16 Holland R. Trends recognised in cases reported to the New South Wales Special Committee investigating deaths under anaesthesia. Anaesth Intensive Care 1987; 15:97-8.

17 Australian Patient Safety Foundation. Crisis Management Manual: COVER $A B C D$ A SWIFT CHECK. Adelaide: Australian Patient Safety Foundation, 1996:74pp. Available at http://www.apsf.net.au/anaesthesia.htm (accessed 6 September 2004). 